\title{
Photochemistry of ketones adsorbed on size/shape selective zeolites. A supramolecular approach to persistent carbon centered radicals
}

\author{
Nicholas J. Turro, ${ }^{*}{ }^{\dagger}$ Ann McDermott, ${ }^{a}$ Xuegong Lei, ${ }^{a}$ Wei Li, ${ }^{a}$ Lloyd Abrams, $^{b}$ M. Francesca Ottaviani, ${ }^{c}$ \\ Hege Støgård Beard, ${ }^{d}$ Kendall N. Houk, ${ }^{e}$ Brett R. Beno ${ }^{e}$ and Patrick S. Lee ${ }^{e}$ \\ a Chemistry Department, Columbia University, New York, NY 10027, USA \\ ${ }^{b}$ E. I. duPont de Nemours, Central Research Department, Experimental Station, Wilmington, DE 19880, USA \\ c University of Florence, Department of Physical Chemistry, Via Gino Capponi, 9, 50121 Florence, Italy \\ ${ }^{d}$ Chemistry Department, University of Oslo, Oslo, Norway \\ e Department of Chemistry and Biochemistry, University of California, Los Angeles, CA 90095-1569, USA
}

${ }^{2} \mathrm{H}$ NMR, EPR, computational and product analyses of the photolysis of 2,4-diphenylpentan-3-one (DPP) adsorbed on MFI size/shape selective zeolites are consistent with supramolecular structural changes as a function of surface coverage that provide a novel method for the generation of persistent diffusing organic free radicals.

The MFI topology zeolites (e.g. silicalite and ZSM-5) are of technical significance in both size/shape selective catalysis and in molecular sieve separations. ${ }^{1}$ The technically attractive properties of MFI zeolites originate from supramolecular, structural (substrates as guests, zeolite crystal as host) and dynamic (shape/size dependent supramolecular molecular diffusion) characteristics, i.e. substrates possess 'effective' molecular diameters comparable to the size/shape of the holes on the external surface of the zeolite crystal and/or the channels and intersections on the internal surface. ${ }^{2}$ The siting and diffusion of a substrate are vital in determining the product selectivity in catalysis and the resolution efficiency in separations. ${ }^{3}$

Understanding the role of diffusion and siting requires the uncoupling of both supramolecular, structural and dynamic features from the effects of activation at active sites and rearrangements of reactive intermediates. The effects of activation are pronounced at high temperatures with zeolites possessing strongly acidic sites (e.g. HZSM-5).

We report a supramolecular photochemical investigation ${ }^{4}$ at room temperature in which the activation and diffusional/siting effects of zeolite catalysis are uncoupled. Activation of the substrate towards reaction is provided by photochemical methods which (i) excite adsorbed substrates whose sitings and reaction products are directly determined by ${ }^{2} \mathrm{H}$ NMR analysis and (ii) produce persistent $t^{5,6}$ adsorbed carbon-centred radical intermediates whose structure and mobility are directly determined by EPR spectroscopy. Computational methods are employed for (i) analysis of the plausibility of the siting and diffusional processes on the external and internal surfaces, (ii) rationalization of the variation of product distribution as a function of supramolecular structure, and (iii) simulation of the EPR spectra of the persistent supramolecular radical species formed upon photoexcitation of the adsorbed substrates.

In the molecular (solution) photochemistry [eqns. (1)-(3)] of 2 ,4-diphenylpentan-3-one (DPP) $\alpha$-cleavage from $\mathrm{T}_{1}$ produces,

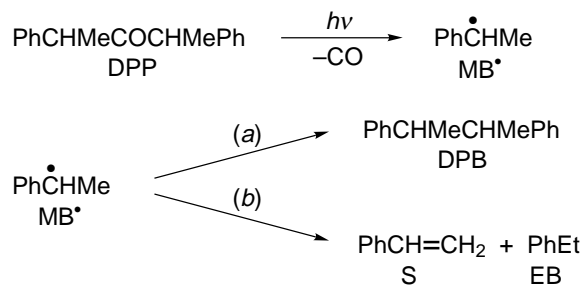

after rapid loss of $\mathrm{CO}, \alpha$-methylbenzyl radicals (MB') [eqn. (1)], which undergo random radical combination to produce 2,3-diphenylbutane (DPB) ( ca. 95\%) and disproportionates to styrene (S) and ethylbenzene (EB) (ca. 5\%). The rates of the radical-radical reactions in eqns. (2) and (3) are close to diffusion controlled ${ }^{5,8}$ and, in the absence of radical scavengers, determine the lifetime of the 'molecular' radicals $\mathrm{MB}^{\text {' }}$.

Results of product analysis and EPR measurements as a function of coverage [DPP/(Na)ZSM-5 (w/w), Si/Al $=80$, average particle size $c a .0 .1 \mu$ ] showed that (i) the dominant products depend on the coverage, with disproportionation to form $\mathrm{S}$ and $\mathrm{EB}$ being favored by low coverage $(<0.3 \%)$ and combination to form DPB being increasingly favored as the coverage increases, ${ }^{9}$ and (ii) the maximum EPR intensity of the signal of the persistent $\mathrm{MB}^{*}$ radicals is insensitive to loading. For example, the ratio of disproportionation to combination of $\mathrm{MB}^{\bullet}$ radicals in solution is typically of the order of 0.1 ; at $0.3 \%$ loading of DPP the ratio is 3.1 .

The values of the half-widths $\left(\Delta H_{1 / 2}\right)$ of the ${ }^{2} \mathrm{H}$ NMR spectra of $\mathrm{C}_{6} \mathrm{D}_{5} \mathrm{CHMeCOCHMeC}{ }_{6} \mathrm{D}_{5}\left(\left[{ }^{2} \mathrm{H}_{10}\right]-\mathrm{DPP}\right)$ as a function of coverage are shown in Fig. 1. These provide information ${ }^{10}$ concerning both the supramolecular structure and dynamics of the $\left[{ }^{2} \mathrm{H}_{10}\right]-D P P / Z S M-5$ system as a function of coverage: (i) a 'sharp' signal $(<1 \mathrm{kHz})$ indicates rapid, isotropic motion and the lack of constraints of the molecular structure of $\left[{ }^{2} \mathrm{H}_{10}\right]$-DPP by the host ZSM-5 structure, i.e. the system is essentially molecular (very weak intermolecular bonds between guest and host); (ii) a 'relatively broad' signal ( $\mathrm{ca} .40 \mathrm{kHz})$ indicates an anisotropic and partial constraint of a weakly bonded supramolecular structure of a DPP/ZSM-5 system; (iii) a 'limiting broad' signal ( $\mathrm{ca} .130 \mathrm{kHz}$ ) indicates relatively strong intermolecular bonds which result in inhibition of motion of a supramolecular $\left[{ }^{2} \mathrm{H}_{10}\right]-D P P / Z S M-5$ system. If the ketone were located on the flat part of the external surface, its motion would be essentially isotropic, and $\Delta H_{1 / 2}$ would be expected to be of the

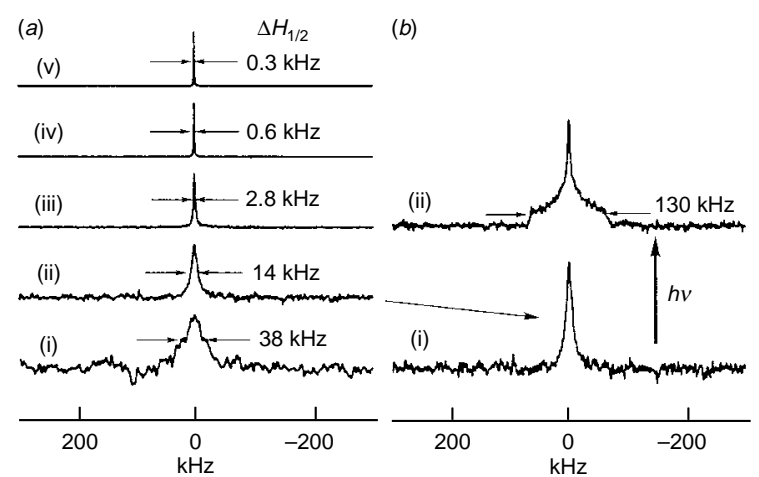

Fig. 1 (a) ${ }^{2} \mathrm{H}$ NMR spectra of ([$\left.{ }^{2} \mathrm{H}_{10}\right]$-DPP) at (i) 0.3 , (ii) 1 , (iii), 2, (iv) 5 and (v) $10 \mathrm{wt} \%$ loading on zeolite. (b) Result of $1 \%$ loading sample (i) before and (ii) after photolysis. 
order of $1 \mathrm{kHz}$. If the ketone were located on the inside of the zeolite $\Delta H_{1 / 2}$ would be of the order of $130 \mathrm{kHz}$. The observed value of $40 \mathrm{kHz}$ is consistent with the ketone being moderately constrained in the holes on the external surface.

Before photolysis (Fig. 1), the ${ }^{2} \mathrm{H}$ NMR spectrum of the DPP/ ZSM-5 system at low loading ( $<1 \%$ loading) exhibits a half width of $c a .40 \mathrm{kHz}$, characteristic of a moderately constrained $\left[{ }^{2} \mathrm{H}_{10}\right]-D P P$ molecule. Thus, we conclude that the DPP molecules at low coverage are adsorbed in the holes at the interface of the external and internal surfaces of the ZSM-5 crystal. Photolysis of $\left[{ }^{2} \mathrm{H}_{10}\right]$-DPP at room temperature results in a change in the ${ }^{2} \mathrm{H}$ NMR spectrum and the appearance of a new broad feature ( $c a .130 \mathrm{kHz}$ ) which grows as the extent of photolysis increases (Fig. 2).

For high coverage before photolysis the spectrum shows mainly a sharp peak $\left(\Delta H_{1 / 2}<1 \mathrm{kHz}\right)$, with the broad peak being buried in the base line due to the $\left[{ }^{2} \mathrm{H}_{10}\right]$-DPP molecules in the holes. We conclude that photolysis of $\left[{ }^{2} \mathrm{H}_{10}\right]$-DPP at high coverage does not lead to a significant increase of the broad peak because most of the photolyzed $\left[{ }^{2} \mathrm{H}_{10}\right]$-DPP molecules occur in multilayers at high coverage, and the radicals produced in these fluid multilayers are very mobile and undergo random radical-radical combination [eqn. (2)]. This view is supported by the fact that the major product of photolysis at high coverage is that expected from 'molecular' or solution conditions, i.e. DPB ( $c a .95 \%)$; most of the absorbed light excites the large excess of ketones in the multilayers rather than the relatively smaller number adsorbed in the holes at the interface.

The steady state photolysis of DPP adsorbed on ZSM-5 produced intense, long lived (many hours) EPR signals at all coverages studied. The observed spectrum fits a simulation ${ }^{12}$ of a powder spectrum of $\mathrm{MB}^{*}$ as expected from the restricted mobility of $\mathrm{MB}^{*}$ adsorption on the internal surface. Computational analysis indicates that the lowest energy supramolecular structure of $\mathrm{MB}^{\cdot}$ has the $\mathrm{Ph}$ group placed in an intersection between the channels, and the alkyl radical moiety placed in a channel between the intersections. This supramolecular geometry possesses a substantial barrier to motion, but allows the achievement of a roughly planar structure at the radical center. The hyperfine coupling constants computed and determined experimentally, ${ }^{11,12}$ taking into account anisotropies due to restricted motion, are sufficient for the simulation. Finally, computations were made of the external surface area available for adsorption of DPP in order to estimate the relationship between the macroscopic composition (loading w/w) and the surface coverage. The measured external surface area of the ZSM-5 sample 9 is of the order of $16 \mathrm{~m}^{2} \mathrm{~g}^{-1}$, which, when compared to the computations, implies that a monolayer of DPP will be formed when the coverage is $c a .0 .4 \%$, the coverage at which the experimentally observed salient effects on products and spectroscopic properties begin to change. As the coverage increases to values of $c a .1 \%$ and greater, the results become characteristic of a molecular, two dimensional film rather than of an adsorbed layer, because the bulk of the ketone molecules are in the multilayer and not adsorbed on the zeolite external surface. As the coverage increases, the lifetime of the radicals decreases and the ratio of disproportionation to combination decreases, i.e. the product distribution becomes more like that in homogeneous solution. These results are also consistent with the formation of multilayers of ketones on the external surface, so that ketone molecules finds themselves increasingly in a 'two-dimensional' liquid as the loading increases, and the results tend toward those for homogeneous solutions.

The persistence, ${ }^{6,7}$ or lifetime, of $\mathrm{MB}^{*}$ is limited by either (i) the diffusion of two $\mathrm{MB}^{*}$ radicals into the vicinity of an intersection, or (ii) disproportionation, the lowest energy supramolecular reaction of the system within the intersection. Thus, as a consequence of their supramolecular structure, two


favored' radical-radical combination, but instead undergo disproportionation to S and EB [eqn. (3)]. The structural basis for this selectivity may be either a supramolecular 'steric' or 'dynamic' effect. The steric effect would result from the high energy required for a $\mathrm{C}-\mathrm{C}$ bond to form $\mathrm{DPB}$ at an intersection, as indicated by computation, and resulting from the supramolecular steric effects associated with the compression of a DPB molecule into the limited space available in the vicinity of the intersections and channels. The less sterically demanding radical-radical disproportionation in the vicinity of the intersections becomes the default supramolecularly-allowed radical-radical reaction, but even this reaction is still remarkably slow and allows the supramolecular MB $/$ ZSM-5 radicals to become persistent for hours at room temperature. A dynamic mechanism, termed a diffusional 'maze' effect, could also cause the persistence of otherwise reactive radicals. In the maze effect, the molecular traffic patterns of the radicals lead to rate limiting infrequent encounters in the vicinity of the intersection. In the extreme form of the maze effect an encounter leads to a 'diffusion controlled' reaction.

The ${ }^{2} \mathrm{H}$ NMR, EPR, computational and photochemical product analyses are all supported by the same supramolecular structural and dynamic interpretation of the results at high and low coverages. The supramolecular photochemistry of DPP adsorbed on ZSM-5 molecular sieve zeolites depends dramatically on the composition of the DPP/ZSM-5 system, because the supramolecular constitutional structure (connectivity relationship of the guest and host structures) depends on the system's composition. At low loading ( $<0.3 \%)$ DPP is mainly adsorbed in the holes on the external surfaces that provide access to the internal surface. At intermediate loadings ( $c a .1 \%)$, when the limited amount of holes is plugged with DPP molecules, as the coverage increases, the external framework surface between the holes becomes covered with DPP molecules until a monolayer is formed. At 'high' loadings ( $>1 \%$ ), both the holes and the framework's external surface are covered (a monolayer is formed) so that, as the coverage increases, multilayers or two dimensional films of DPP are formed on the external surface.

The authors thank Dr Paul Krusic (DuPont), Professors Hans Fischer and Henning Paul for enlightening discussions, and the NSF for financial support. X. G. L. and W. L. thank the Kanagawa Academy of Science and Technology for funding.

\section{Notes and References}

$\dagger$ E-mail: turro@chem.columbia.edu

1 See, for example, R. M. Dessau, Selective Sorption Properties of Zeolties, ACS Symp. Ser., 1980, 135, 123.

2 V. R. Choudhary, V. S. Nayak and T. V. Chaudhary, Ind. Eng. Chem. Res., 1997, 36, 1812

3 P. B. Weisz, Pure Appl. Chem., 1980, 52, 2091; P. B. Weisz, Ind. Eng. Chem. Fundam., 1986, 25, 53.

4 For a review of the photochemistry of organic molecules adsorbed on zeolites, see N. J. Turro, Pure Appl. Chem., 1986, 58, 1219.

5 (a) D. Griller and K. U. Ingold, Acc. Chem. Res., 1976, 9, 13; (b) G. D. Mendenhall and K. U. Ingold, J. Am. Chem. Soc., 1973, 95, 3422 .

6 Self reaction via combination or disproportionation determines the persistence of radicals inert to rearrangement and reaction with the environment.

7 N. D. Ghatlia and N. J. Turro, J. Photochem. Photobiol. A: Chem., 1991, 57, 7; B. H. Baretz and N. J. Turro, J. Am. Chem. Soc., 1983, 105, 1309.

8 H. Fischer, J. Am. Chem. Soc., 1986, 108, 3925 and references therein.

9 The surface area of the ZSM-5 samples was determined to be $c a$. 15 $\mathrm{m}^{2} \mathrm{~g}^{-1}$ by mercury porosimetry.

10 For a discussion of the relationship between motion and line shape or ${ }^{2} \mathrm{H}$ NMR spectroscopy, see R. J. Wittebort, E. T. Olejniczak and R. G. Griffin, J. Chem. Phys., 1987, 86, 5411

11 R. Batra, B. Giese, B. M. Spichty, G. Gescheidt and K. N. Houk, J. Phys. Chem., 1996, 100, 1837.

12 M. S. Conradi, H. Zeldes and R. Livingston, J. Phys. Chem., 1979, 83, 633.

Received in Corvallis, OR, USA, 1st December 1997; 7/08691A 